\title{
Comparative Effectiveness of Pneumococcal Vaccination With PPV23 and PCV13 in COPD Patients Over a 5-Year Follow-Up Cohort Study
}

\section{Galina L. Ignatova}

South Ural state medical University

\section{Sergey N. Avdeev}

Sechenov University

Vladimir Nikolayevich Antonov ( $\nabla$ vladimir.antonov.SUSMU@mail.ru )

South Ural state medical University

\section{Research Article}

Keywords: chronic obstructive pulmonary disease, PCV13, PPV23, pneumonia

Posted Date: April 23rd, 2021

DOI: https://doi.org/10.21203/rs.3.rs-445382/v1

License: (c) (i) This work is licensed under a Creative Commons Attribution 4.0 International License. Read Full License

Version of Record: A version of this preprint was published at Scientific Reports on August 5th, 2021. See the published version at https://doi.org/10.1038/s41598-021-95129-w. 


\section{Abstract}

\section{Background}

Vaccination against Streptococcus pneumoniae is among the most effective measures for preventing pneumonia and reducing the rate of chronic obstructive pulmonary disease (COPD) exacerbations. The objective of this work was to evaluate the long-term effectiveness of PCV13 and PPV23 for preventing pneumonia and COPD exacerbations.

\section{Methods}

The open-label, prospective, observational cohort study involved 302 male patients aged $\geq 45$ years: PCV13 group ( $n=123)$; PPV23 group ( $n=32)$; and vaccine-naïve group $(n=147)$. The primary endpoint included the frequency of pneumonia episodes and COPD exacerbations per year over a 5-year follow-up period. The secondary endpoints included the dynamics of dyspnea severity (MMRC), the BODE index, FEV1, the CAT index, the SGRQ score, and the results of 6-min walk test.

\section{Results}

Vaccination with PCV13 and PPV23 significantly reduces the total rate of pneumonia during the first year after vaccination. Starting with the second year, clinical effectiveness in PPV23 group decreases compared with both PCV13 group and vaccine-naïve patients. Pneumonia by year 5 after vaccination were registered in versus $47 \%$ of patients in the PPV23 group $3.3 \%$ of patients in the PCV13 group ( $p<0.001)$; COPD exacerbations - in $81.3 \%$ versus $23.6 \%$, respectively $(p<0.001)$. Vaccination with PCV13 significantly reduced and maintained the BODE index over the 5-year follow-up period.

\section{Conclusion}

Although both vaccines have comparable clinical effects during the first year after vaccination, only PCV13 is characterized by persistent clinical effectiveness during the 5-year follow-up period. Patients older than 55 years on PPV23 have significantly higher risks of having pneumonia episodes more frequently during the long-term follow-up.

\section{Introduction}

Chronic obstructive pulmonary disease (COPD) is a common disorder characterized by ongoing respiratory symptoms and airflow obstruction caused by airway and/or alveolar abnormalities typically emerging after substantial exposure to hazardous particles and gases (1). Although COPD is preventable and treatable, its periodic exacerbations considerably worsen patients' quality of life and increase the risks of unfavorable outcomes, morbidity, and mortality.

Frequent exacerbations are among the key factors of poor prognosis of COPD in patients without comorbidities (2). Each new exacerbation of COPD aggravates the disease course. An exacerbation abruptly increases the risks of developing life-threatening complications and irreversible changes in the 
respiratory system, which reduce the forced expiratory volume (FEV) and worsen clinical symptoms. Influenza and streptococcus pneumonia infections are among the most common reasons for exacerbation; they worsen the overall prognosis of the disease $(3,4)$. Remaining an active smoker is another significant factor inducing disease exacerbations $(5,6)$. Smoking cessation improves the disease prognosis and reduces the risks of unfavorable outcomes and frequent exacerbations by $25 \%$. However, in real-life practice, a significant percentage of patients continue smoking in spite of the physician's recommendations. For this very reason, prevention of COPD exacerbations is the key factor for rehabilitation for these patients. Pulmonary rehabilitation is the key parameter of treatment effectiveness, since it is associated with improving clinical symptoms, quality of life, as well as patients' physical and emotional status.

The Global Initiative for Chronic Obstructive Lung Disease (GOLD) recommends influenza vaccination at evidence $A$ level due to its ability to reduce hospitalization and mortality rates, and pneumococcal vaccination at evidence B level to prevent community-acquired pneumonia(1). Nevertheless, a specific vaccination protocol, duration, and persistence of the effect depending on patient's characteristics still need to be elucidated. Vaccines of two types are currently used to prevent pneumococcal infections: 13valent conjugate and 23-valent polysaccharide ones. The question regarding the effectiveness and the personalized approach to vaccine selection is rather relevant. On the one hand, the 23-valent vaccine with a wide range of serotypes allows one to broaden its spectrum of activity, especially in the regions endemic for these serotypes. On the other hand, the immune responses induced by polyvalent and conjugate vaccines differ fundamentally. Conjugate vaccines proved to have a prolonged effect and persistent clinical effectiveness in all age groups. Furthermore, the long-term effect of using polyvalent vaccines (especially for immunocompromised patients) and the sequelae for the immune system have been insufficiently studied. It was demonstrated for COPD patients that PPV23 significantly reduces the pneumonia incidence rate over a 3-year period in COPD patients younger than 65 years, in patients with severe respiratory disturbance (predicted FEV1 $<40 \%$ ), and those having cardiovascular comorbidities(4). However, only the overall effectiveness in reducing the risks of developing pneumonia in elderly patients (above 65 years) and COPD patients has been demonstrated for PCV13 (7). Individual factors responsible for the high or low clinical effectiveness of vaccination for COPD patients have not been studied.

The question regarding the effectiveness of co-immunization with vaccines against Streptococcus pneumoniae + influenza virus still remains disputable $(8,9)$. Some studies have demonstrated that upon co-immunization, the positive effect on cardiovascular mortality is more likely to be associated with the influenza vaccine rather the pneumococcal one. However, only the polysaccharide pneumococcal vaccine was often utilized in these studies.

To ensure personalized selection of the maximally effective vaccination protocol and stratify the risk groups of COPD exacerbation and recurrent pneumonia, it is necessary to compare patients vaccinated using different protocols. The long-term effects should be evaluated not only between the groups, but also with the vaccine-naïve control group having the same risk factors. Therefore, the objective of our study was to compare the effectiveness of vaccination with PPV23 and PCV13 to prevent COPD exacerbation and recurrent pneumonia episodes during a 5-year period after vaccination, as well as to compare the 
vaccination effectiveness to the data in the vaccine-naïve control group characterized by the same baseline disease severity and risk factors.

\section{Methods}

\section{Study design and patient selection}

This was a prospective observational cohort study involving patients subjected to routine vaccination between October and December 2012. Male patients diagnosed with COPD (aged $\geq 45$ years) followed up at the Chelyabinsk Municipal Center of Pulmonology. COPD was diagnosed according to the GOLD 2011 criteria. All patients were receiving standard treatment according to the GOLD 2011 guidelines (10). The patients were allocated into three groups: the patients vaccinated with the 23-valent vaccine (PPV23 group), those vaccinated with the 13-valent vaccine (PCV13 group), and vaccine-naïve patients (control group). Follow-up duration was 5 years; the key control parameters were evaluated annually. The inclusion criteria were as follows:

- Male patients aged $\geq 45$ years, inclusive.

- Clinically verified diagnosis of COPD made in accordance with the GOLD 2011 guidelines.

- Current or past smokers with $\geq 10$ pack-years history of smoking (e.g., at least one cigarette pack per day for 10 years or an equivalent value).

- The FEV1/FVC ratio after inhaling bronchodilator $(400 \mu \mathrm{g}$ salbutamol) is $<0.70$.

- The patient has undergone spirometry in compliance with the guidelines of the American Thoracic Society (ATS) and European Respiratory Society (ERS) (2005), and reproducible results were obtained.

- The patient is willing and able to attend control visits and undergo all the scheduled analyses and procedures.

Exclusion criteria:

- The presence of a clinically relevant respiratory comorbid condition along with COPD (e.g., bronchial asthma, tuberculosis, bronchiectasis, or other nonspecific lung diseases).

- Severe comorbid conditions.

- The presence of malignant tumors of any organ (either treated or not) in the medical history over the past five years

- Daily oxygen therapy (>12 hrs per day).

- Administration of systemic corticosteroids within the past three months.

\section{Ethics}

The study was conducted according to the Good Clinical Practice standards, which ensure that the design, implementation, and communication of data are reliable, that patients' rights are protected, and that the integrity of subjects is maintained by the confidentiality of their data. The study was approved by the local 
ethics committee of the Regional Clinical Hospital \#4 (protocol 8. 21.10.2012, LIEC of State-Financed Healthcare Institution, Chelyabinsk Regional Clinical Hospital). All patients provided written informed consent in accordance with the Declaration of Helsinki, which included their consent for using their data in analyses and to be presented.

\section{Data collection}

The results of clinical and instrumental analyses (pulse oximetry, spirometry, whole-body plethysmography, and 6-min walk test) were collected at enrollment and then every year. The dyspnea severity was evaluated using the Modified Medical Research Council dyspnea scale (MMRC) (11) as grade $0-4$. Vaccination effectiveness was assessed according to the number of recurrent pneumonia episodes, the number of COPD exacerbations, and the number of hospitalizations because of disease exacerbation.

\section{Statistical Analysis}

The calculations were performed using the R Statistical Package (http://www.r-project.org). Descriptive statistics were shown as absolute frequencies or medians with interquartile range. The Mann-Whitney Utest, or ANOVA, or Pearson's $\chi^{2}$ test, or Fisher's exact test and non-parametric Kruskal-Wallis test by rank and median multiple comparisons were used depending on type of the analyzed data.

All the reported $p$ values were based on two-tailed tests of significance; the $p$ values $<0.05$ were regarded as statistically significant. The STATISTICA 7.0 software (StatSoft, USA) and RStudio software version 1.0.136 (Free Software Foundation, Inc., USA) with R packages version 3.3.1 (R Foundation for Statistical Computing, Austria) were used for the analyses.

\section{Results}

\section{Baseline characteristics}

Over the period between October and December 2012, 150 patients were vaccinated with PCV13; 32 patients, with PPV23. The study was included a control group consisting of 212 vaccine-naïve patients. After the adjustment in relation to age, dyspnea severity, and duration of COPD, 123 patients vaccinated with PCV13, 32 patients vaccinated with PPV23, and 147 patients who had refused vaccination for various reasons were enrolled. Table 1 summarizes the overall characteristics of patients at baseline. 
Table 1

Baseline demographic and clinical characteristics of patients enrolled in the study.

\begin{tabular}{|c|c|c|c|c|}
\hline Parameter & $\begin{array}{l}\text { No } \\
\text { vaccination } \\
(n=147)\end{array}$ & $\begin{array}{l}\text { PPV23 } \\
(n=32)\end{array}$ & $\begin{array}{l}\text { PCV13 } \\
(n=123)\end{array}$ & $\begin{array}{l}p \\
\text { value }\end{array}$ \\
\hline \multicolumn{5}{|l|}{ Demography and anamnesis } \\
\hline Age (years) & $63(60: 66)$ & $\begin{array}{l}60(55: \\
64.25)\end{array}$ & $\begin{array}{l}61(57: \\
66)\end{array}$ & 0.133 \\
\hline Aged $>65$ years & $56[38.1 \%]$ & 8 [25\%] & $\begin{array}{l}36 \\
{[29.27 \%]}\end{array}$ & 0.181 \\
\hline Age of COPD onset & $6(4: 7)$ & $\begin{array}{l}6(4: \\
6.25)\end{array}$ & $5(2: 8)$ & 0.103 \\
\hline BMI (at baseline) (imt.0y ) & $21(19: 23)$ & $\begin{array}{l}21(19: \\
23.25)\end{array}$ & $\begin{array}{l}21(19: \\
23)\end{array}$ & 0.387 \\
\hline \multicolumn{5}{|l|}{ COPD characteristics } \\
\hline CAT (at baseline) ( cat.0y ) & $\begin{array}{l}25(21.5: \\
30)\end{array}$ & $\begin{array}{l}24(21.8 \\
: 30.3)\end{array}$ & $\begin{array}{l}25(21: \\
30.5)\end{array}$ & 0.924 \\
\hline MMRC: & 19 [12.93\%] & 6 & 12 & 0.219 \\
\hline grade 2 & $76[51.7 \%]$ & \multirow{2}{*}{$\begin{array}{l}18 \\
{[56.25 \%]}\end{array}$} & \multirow{2}{*}{$\begin{array}{l}56 \\
{[45.53 \%]}\end{array}$} & \\
\hline grade 3 & 52 [35.37\%] & & & \\
\hline grade 4 & & 8 [25\%] & $\begin{array}{l}55 \\
{[44.72 \%]}\end{array}$ & \\
\hline BODE index & $5(4: 6)$ & $\begin{array}{l}5(4: \\
5.25)\end{array}$ & $5(4: 6)$ & 0.193 \\
\hline 6MWT (at baseline) & $\begin{array}{l}370(318: \\
456)\end{array}$ & $\begin{array}{l}390(313 \\
: 497)\end{array}$ & $\begin{array}{l}370(340 \\
: 425)\end{array}$ & 0.453 \\
\hline SGRQ (Summary) & $39(35: 47)$ & $\begin{array}{l}37.5(33 \\
: 47)\end{array}$ & $\begin{array}{l}38(35: \\
46)\end{array}$ & 0.477 \\
\hline Exacerbations over the year preceding vaccination: & $24[16.33 \%]$ & $\begin{array}{l}7 \\
{[21.88 \%]}\end{array}$ & $\begin{array}{l}22 \\
\text { [17.89\%] }\end{array}$ & 0.619 \\
\hline None & $\begin{array}{l}122 \\
{[82.99 \%]}\end{array}$ & 25 & 98 & \\
\hline One exacerbation & $1[0$ 68\%] & [78.12\%] & [79.67\%] & \\
\hline Two exacerbations & & 0 [0\%] & $\begin{array}{l}3 \\
{[2.44 \%]}\end{array}$ & \\
\hline
\end{tabular}




\begin{tabular}{|c|c|c|c|c|}
\hline Parameter & $\begin{array}{l}\text { No } \\
\text { vaccination } \\
(n=147)\end{array}$ & $\begin{array}{l}\text { PPV23 } \\
(n=32)\end{array}$ & $\begin{array}{l}\text { PCV13 } \\
(n=123)\end{array}$ & $\begin{array}{l}p \\
\text { value }\end{array}$ \\
\hline \multirow{2}{*}{$\begin{array}{l}\text { Hospitalizations for the exacerbation over the year } \\
\text { preceding vaccination: }\end{array}$} & 13 [8.84\%] & \multirow{2}{*}{$\begin{array}{l}4 \\
{[12.5 \%]}\end{array}$} & \multirow{2}{*}{$\begin{array}{l}10 \\
{[8.13 \%]}\end{array}$} & \multirow[t]{5}{*}{0.625} \\
\hline & 133 & & & \\
\hline None & [90.48\%] & & $\begin{array}{l}110 \\
{[89.43 \%]}\end{array}$ & \\
\hline One hospitalization & \multirow{2}{*}{$1[0.68 \%]$} & ] & & \\
\hline Two hospitalizations & & [010] & [2.44\%] & \\
\hline \multicolumn{5}{|l|}{ Pneumonia episodes } \\
\hline $\begin{array}{l}\text { Pneumonia episodes over the year preceding } \\
\text { vaccination, total: }\end{array}$ & $\begin{array}{l}120 \\
{[81.63 \%]}\end{array}$ & 24 [75\%] & $\begin{array}{l}104 \\
{[84.55 \%]}\end{array}$ & \multirow[t]{4}{*}{0.492} \\
\hline None & 27 [18.37\%] & 8 [25\%] & 18 & \\
\hline One pneumonia & \multirow[t]{3}{*}{$0[0 \%]$} & & & \\
\hline \multirow[t]{2}{*}{ Two pneumonias } & & & [0.81\%] & \\
\hline & & \multicolumn{2}{|l|}{$0[0 \%]$} & \\
\hline $\begin{array}{l}\text { Pneumonia episodes requiring hospitalization over the } \\
\text { year preceding vaccination: }\end{array}$ & $\begin{array}{l}120 \\
{[81.63 \%]}\end{array}$ & $\begin{array}{l}27 \\
{[84.38 \%]}\end{array}$ & $\begin{array}{l}104 \\
{[84.55 \%]}\end{array}$ & \multirow[t]{4}{*}{0.715} \\
\hline None & 27 [18.37\%] & 5 & 18 & \\
\hline One hospitalization with pneumonia & \multirow[t]{2}{*}{$0[0 \%]$} & \multirow{2}{*}{$0[0 \%]$} & \multirow{2}{*}{$\begin{array}{l}1 \\
{[0.81 \%]}\end{array}$} & \\
\hline Two hospitalizations with pneumonia & & & & \\
\hline
\end{tabular}

The patients were matched for age, duration of COPD, and severity of clinical signs of COPD. After the adjustment, each group contained patients with MMRC $\geq 2$. Most of the enrolled patients ( $82.5 \%)$ had at least one COPD exacerbation during the year preceding the enrollment. At least one pneumonia episode was reported by $17.9 \%$ of patients during the preceding year.

\section{Effectiveness of vaccination in preventing pneumonia}

Each patient was followed up for 5 years after the baseline characteristics had been collected. The pneumonia rate was comparable at baseline and significantly decreased in both vaccinated groups compared to vaccine-naïve patients (Fig. 1). The percentage of patients having at least one pneumonia episode reduced to $4.9 \%$ in the PCV13 group and to $6.3 \%$ in the PPV23 group versus $15 \%$ level in the vaccine-naïve patients ( $p=0.024$ and $p=0.707$, respectively). However, patients in the PPV23 group showed significantly worse results during the second year after the vaccination even compared to those in the control (vaccine-naïve) group, and reached the pneumonia rate of $47 \%$ by year 5 after the vaccination (as compared with $23.1 \%$ in control group without vaccination, $p=0.027$ ). 


\section{Effectiveness of vaccination for reducing the rate of COPD exacerbations}

Both types of vaccines were strongly associated with significant reducing of COPD exacerbation rate as compared to the control group (Fig. 2). However, in the PPV23 group, this effect was gradually declining after year 1 post-vaccination and reached the level comparable to that for vaccine-naïve patients by year 5 post-vaccination.

Similar dynamics were observed for the hospitalization frequency (Fig. 3). At baseline, the overall percentage of patients having at least one hospitalization per year was $90.2 \%$. Vaccination had a significant effect during the first year of the follow-up: only $18.8 \%$ of PPV23 patients and $16.3 \%$ of PCV13 patients were hospitalized. In the PCV13 group, this effect persisted during the entire follow-up period, and the percentage of patients having disease exacerbation over the 5-year period was $\leq 23 \%$. In the PPV23 group, this effect was significantly reduced after the 2 -year follow-up: the exacerbation rate was 75,88, and $97 \%$ during the third, fourth, and fifth years of the follow-up, respectively.

\section{Effectiveness of vaccination for improving patients' quality of life and respiratory function parameters}

Assessment of the comprehensive effect of vaccination protocol or the absence of vaccination on the BODE (a multidimensional grading system for evaluating the health status of patients) index(12) demonstrated that although both utilized vaccines significantly reduced the mortality rate during the first two years after vaccination, only the PCV13 vaccine provided a persistent effect for three years or longer (Fig. 4). The BODE score was reduced by one or more points one year after the vaccination in 30 (93.8\%) PPV23 patients, 123 (100\%) PCV13 patients, and only $75(51 \%)$ vaccine-naïve patients $(p<0.0001)$. However, the BODE score has been increasing significantly in PPV23 group for the following 4 years. At the end of follow-up period it was $5(4: 5)$ in PPV23 group and $5(4: 6)$ in no vaccination group $(p>0.999)$. In the PCV13 group BODE score remains low with $3(2: 3)$ points after 5 years $(p<0.0001$ as compared with both PPV23 group and no vaccination group).

Dyspnea severity by MMRC in patients was monitored during the entire follow-up period (Fig. 5). At baseline, the groups were comparable in terms of MMRC $(p=0.219)$; patients having grade 3 predominated in all the groups (52\% in the vaccine-naïve group; 56\% in the PPV23 group; and 46\% in the PCV13 group). A smaller percentage of patients had grade 4 , and the fewest number of patients had grade 2 . However, as early as one year after vaccination, the groups significantly differed in terms of dyspnea severity. In all vaccinated patients (even those who had grade 4 dyspnea at baseline), MMRC decreased to grade 3 or even grade 2; this effect persisted throughout the entire follow-up period. Even in the vaccine-naïve group, the percentage of patients having grade 4 dyspnea decreased during the first year after the vaccination. The overall improvement in health status of all patients during the first year could have resulted from the fact that patients were visiting the physician on a regular basis and, therefore, were more compliant to the therapy received. Nevertheless, this trend towards improvement was maintained only in the PCV13 group. 
Moreover, the respiratory function parameters were worsened in the PPV23 group down to the level lower than the baseline level: none of the patients had grade 1 by the end of the follow-up period, while grade 2 was observed in only one (3.1\%) patient.

Assessment of the dynamics of other clinical and laboratory signs showed that there was an overall trend towards improvement of the health status of vaccinated patients compared to vaccine-naïve ones during the first year in terms of such parameters as FEV1, CAT, and 6-min walk test results (Fig. 6). During the following 4 years, the clinical effect persisted only in the PCV13 group, while patients' condition was significantly worsened in the PPV23 group, and parameters comparable to those in the vaccine-naïve group were reached by year 5 .

\section{Factors associated with favorable clinical outcomes}

Assessing the likelihood of reduced rate of pneumonia episodes (or their absence) showed that two main factors associated with favorable outcomes included vaccination with PCV13 and age at vaccination (Fig. 7). The vaccine type was a much more important factor compared to the baseline FEV1, which had no substantial effect on patients' prognosis. An analysis of the risk model showed that regardless of the age of the patient or FEV1 at the moment of vaccination, PCV13 could significantly reduce the frequency of pneumonia or completely prevent it in more than $95 \%$ of patients for up to 5 years period. At the same time, the use of PPV23 is effective only for patients younger than 55 years and has a limitation on the duration of the effect.

\section{Discussion}

Comparison of clinical effectiveness of vaccines for preventing pneumonia, as well as their mediated effect on the health status of COPD patients, demonstrated that both PCV13 and PPV23 are effective during the first 1-2 years after the vaccination. However, the effectiveness of PPV23 decreases noticeably over time: PPV23 ensured proper protection only within two years after vaccination, while PCV13 exhibited a high level of protection during the entire follow-up. Furthermore, the risk of complications in patients vaccinated with PPV23 increased after 3 years after the vaccination compared to vaccine-naïve patients.

Vaccination effectiveness directly depends on various factors, such as the serotypic pattern, patients' age, and the health status at baseline. Many researchers have mentioned the positive effect of vaccination on the outcomes (13) and cost effectiveness of vaccination (14) for PPV23 and PCV13 (7). However, the follow-up period was limited (3 years after the vaccination) for most studies where the vaccines were compared directly. This fact does not allow one to take into account the effect of the decline in effectiveness of vaccination with PPV23 and PCV13 over time. Hence, the question regarding the long-term vaccination effectiveness still remains open. This question becomes especially relevant in the presence of risk factors, such as smoking, FEV1(15), cardiovascular pathology(16), and other factors $(12,17)$ worsening the prognosis for developing severe forms of pneumonia. In current study, all patients had high risk factors for developing severe pneumonia: age > 45 years; smoking; and dyspnea severity grade assessed using the 
MMRC scale $\geq 2$. Nevertheless, pneumococcal vaccination was shown to effectively prevent pneumonia in these patients.

The previous studies assessing the effectiveness of PPV23 showed that the patients differed in terms of clinical effectiveness of vaccination depending on their age (4). The risk of vaccine failure increased significantly in patients older than 65 years. Our study has confirmed this observation and demonstrated that this effect is valid only for the polysaccharide vaccine. For PCV13, patient's age was not a significant factor affecting vaccine effectiveness. Our study found no evidence for the earlier demonstrated influence of FEV1 on vaccine effectiveness. This was probably because only $2.9 \%$ of patients in our sample had FEV $<40 \%$, which was regarded as the threshold level for this parameter.

Although the primary objective of vaccination is to reduce the risk of pneumonia in patients with COPD, it also indirectly affects the patients' overall health status. The study (7)] demonstrated that vaccination with PCV13 reduced the risk of hospitalization (for any reasons) in stable patients having no exacerbations (non-exacerbators), but not in patients having COPD exacerbations within the previous year. In our study, we found that vaccination with PCV13 was associated with the significant overall decline in the exacerbation and hospitalization rates. Before enrollment, the rate of hospitalization because of COPD exacerbation was $>90 \%$. Administration of any pneumococcal vaccine reduced the exacerbation rate over fourfold during the first year. Hence, pneumococcal vaccination has a positive effect on the course of COPD, which includes reduced exacerbation rate and improved overall quality of life. However, reduced hospitalization rates were stably observed only for PCV13, while the exacerbation rate and the rate of exacerbation-related hospitalizations increased abruptly as early as two years after vaccination with PPV23.

The observed decline in tolerance to pneumonia and the increasing percentage of patients with severe pneumonia in the PPV23 group after three-year follow-up is potentially related to hyporesponsiveness. Polysaccharide vaccines stimulate the short-lived B-cell response as they influence the terminal differentiation of the existing memory B-cells into plasma cells, thus depleting the memory B-cell pool (18). This process is not confined to hyporesponsiveness to subsequent vaccination but causes an overall decline in immune response. In combination with the elderly age and the numerous risk factors in COPD patients, this process increases pneumonia severity and the frequency of pneumonia episodes in patients 3-5 years after vaccination with PPV23 even compared to vaccine-naïve patients.

Our study has a number of limitations. First, it has a non-randomized design due to ethical considerations: only patients having additional administrative reasons and warned about the potential risks were enrolled to form vaccine-naïve groups. In order to minimize the risk of bias associated with the non-randomized study design, we employed the PSM methods to select matching patient groups. Second, the size of patient groups differed significantly. Third, the study involved PP analysis rather than ITT one. Nevertheless, this study was the first study to analyze long-term (5-year) clinical effectiveness of vaccination with PCV13 and PPV23 in patients with COPD. Further trials involving large cohorts and registries will make it possible to assess additional risks associated with the long-term effect of hyporesponsiveness in patients after vaccination with PPV23 and its potential effect on quality of life and the risks of unfavorable outcomes. 


\section{Conclusion}

Pneumococcal vaccination with PCV13 and PPV23 significantly improves the clinical status of COPD patients: there are positive trends for such parameters as the dyspnea rate, exercise tolerance, reduction of the rate of pneumonia episodes and exacerbations of the underlying disease. However, while the positive effect persists over the 5-year follow-up for PCV13, it gradually declines in patients vaccinated with PPV23 starting year 2 after the vaccination. Vaccination with PPV23 was associated with a higher risk of severe pneumonia after 3 years post-vaccination and further on compared to the risk in vaccine-naïve patients.

\section{Abbreviations}

COPD: chronic obstructive pulmonary disease; FEV: forced expiratory volume; GOLD: Chronic Obstructive Lung Disease; ATS: American Thoracic Society; ERS: European Respiratory Society; MMRC: Modified Medical Research Council;

\section{Declarations}

\section{Acknowledgements}

The authors gratefully acknowledge A. Moskalev and A. Alshevskaya for help in biostatistics.

\section{Provenance and peer review}

Not commissioned; externally peer reviewed.

\section{Authors' contributions}

GI and SA and VA were responsible in the literature search, study design,data analysis and interpretation, preparation of figures and/or writing. SA was involved in the data collection, analysis and interpretation and critical revision of the manuscript. VA was involved in the interpretation and writing of the manuscript. $\mathrm{GI}, \mathrm{SA}$, VA were involved in the data collection and critical revision of the manuscript.GI, SA, and VA were involved in the data interpretation and critical revision of the manuscript. All authors read and approved the final manuscript.

The conflict of interest disclosure: The authors declare that there is no conflict of interest regarding the publication of this paper.

The data availability statement: The datasets generated during and/or analysed during the current study are available from the corresponding author on reasonable request.

Funding statement: The author received no specific funding for this article.

\section{Ethics approval and consent to participate}


The study was approved by the local ethics committee of the Regional Clinical Hospital \#4 (protocol 8. 21.10.2012).

\section{References}

1. Alvar A, Decramer M, Frith P. Global Initiative for Chronic Obstructive Lung Disease. Pocket guide to COPD diagnosis, management and prevention. Glob Initiat chronic Obstr lung Dis [Internet]. 2017;142. Available from: https://goldcopd.org/wp-content/uploads/2016/12/wms-GOLD-2017-PocketGuide.pdf

2. Aka Aktürk Ü, Görek Dilektaşlı A, Şengül A, Musaffa Salepçi B, Oktay N, Düger M, et al. Influenza and pneumonia vaccination rates and factors affecting vaccination among patients with chronic obstructive pulmonary disease. Balkan Med J. 2017;34(3):206-11.

3. Shea KM, Edelsberg J, Weycker D, Farkouh R, Strutton D, Pelton S. Rates of Pneumococcal Disease in Adults With Chronic Medical Conditions. Open Forum Infect Dis [Internet]. 2014;1-8. Available from: http://ofid.oxfordjournals.org/content/early/2014/06/01/ofid.ofu038.short

4. Alfageme I, Vazquez R, Reyes N, Muñoz J, Fernández A, Hernandez M, et al. Clinical efficacy of antipneumococcal vaccination in patients with COPD. Thorax. 2006;61(3):189-95.

5. van Eerd EAM, van der Meer RM, van Schayck OCP, Kotz D. Smoking cessation for people with chronic obstructive pulmonary disease. Cochrane Database Syst Rev [Internet]. 2016;(8):1-90. Available from: http://search.ebscohost.com/login.aspx? direct=true\&amp $\% 5 \mathrm{Cndb}=$ cin20\&amp $\% 5 \mathrm{CnAN}=2012162682 \& a m p \% 5 C$ nlang=es\&amp $\% 5 C$ nsite=ehost liv

6. Bhatt SP, Kim Y II, Harrington KF, Hokanson JE, Lutz SM, Cho MH, et al. Smoking duration alone provides stronger risk estimates of chronic obstructive pulmonary disease than pack-years. Thorax. 2018;73(5):414-21.

7. Figueira Gonçalves JM, García Bello MA, Bethencourt Martín N, Díaz Pérez D, Pérez-Méndez LI. Impact of 13-valent pneumococcal conjugate polysaccharide vaccination on severe exacerbations in patients with chronic obstructive pulmonary disease and established cardiovascular disease. Eur J Intern Med. 2019;63(February):e14-6.

8. Schembri S, Morant S, Winter JH, MacDonald TM. Influenza but not pneumococcal vaccination protects against all-cause mortality in patients with COPD. Thorax. 2009;64(7):567-72.

9. Chang YC, Chou YJ, Liu JY, Yeh TF, Huang N. Additive benefits of pneumococcal and influenza vaccines among elderly persons aged 75 years or older in Taiwan - A representative population-based comparative study. J Infect [Internet]. 2012;65(3):231-8. Available from: http://dx.doi.org/10.1016/j.jinf.2012.04.014

10. Vestbo J, Hurd SS, Agustí AG, Jones PW, Vogelmeier C, Anzueto A, et al. Global strategy for the diagnosis, management, and prevention of chronic obstructive pulmonary disease GOLD executive summary. Am J Respir Crit Care Med. 2013;187(4):347-65. 
11. FLETCHER CM. The clinical diagnosis of pulmonary emphysema; an experimental study. Proc R Soc Med. 1952;45(9):577-84.

12. Domenech A, Ardanuy C, Tercero A, García-Somoza D, Santos S, Liñares J. Dynamics of the pneumococcal population causing acute exacerbations in COPD patients in a Barcelona hospital (2009-12): Comparison with 2001-04 and 2005-08 periods. J Antimicrob Chemother. 2014;69(4):9329.

13. Chiou WY, Hung SK, Lai CL, Lin HY, Su YC, Chen YC, et al. Effect of 23-valent pneumococcal polysaccharide vaccine inoculated during anti-cancer treatment period in elderly lung cancer patients on community-acquired pneumonia hospitalization: A nationwide population-based cohort study. Med (United States). 2015;94(26):1-10.

14. Satomura K, Nakahara T, Iwanaga S, Noami M, Kusaka K, Harano K. Effect of 23-valent pneumococcal polysaccharide vaccine on medical expenses in Japan. BMC Health Serv Res. 2014;14(S2):6963.

15. Cuthbert JJ, Kearsley JW, Kazmi S, Kallvikbakka-Bennett A, Weston J, Davis J, et al. The impact of heart failure and chronic obstructive pulmonary disease on mortality in patients presenting with breathlessness. Clin Res Cardiol [Internet]. 2019;108(2):185-93. Available from: http://dx.doi.org/10.1007/s00392-018-1342-z

16. Ignatova GL, Antonov VN. Efficiency of vaccine prophylaxis concentrated pneumococcal vaccine in patients with chronic obstructive lung disease and chronic heart failure. Ter Arkh. 2018;90(8):53-62.

17. Ignatova GL, Antonov VN. Analysis of compliance dynamics in patients with chronic obstructive pulmonary disease on the background of vaccination against pneumococcal infection. Ter Arkh. 2018;90(3):47-52.

18. Pollard AJ, Perrett KP, Beverley PC. Box 1 | polysaccharide and protein-polysaccharide conjugate vaccines. 2009;426(2000):422-6. Available from: www.nature.com/reviews/immunol

\section{Figures}




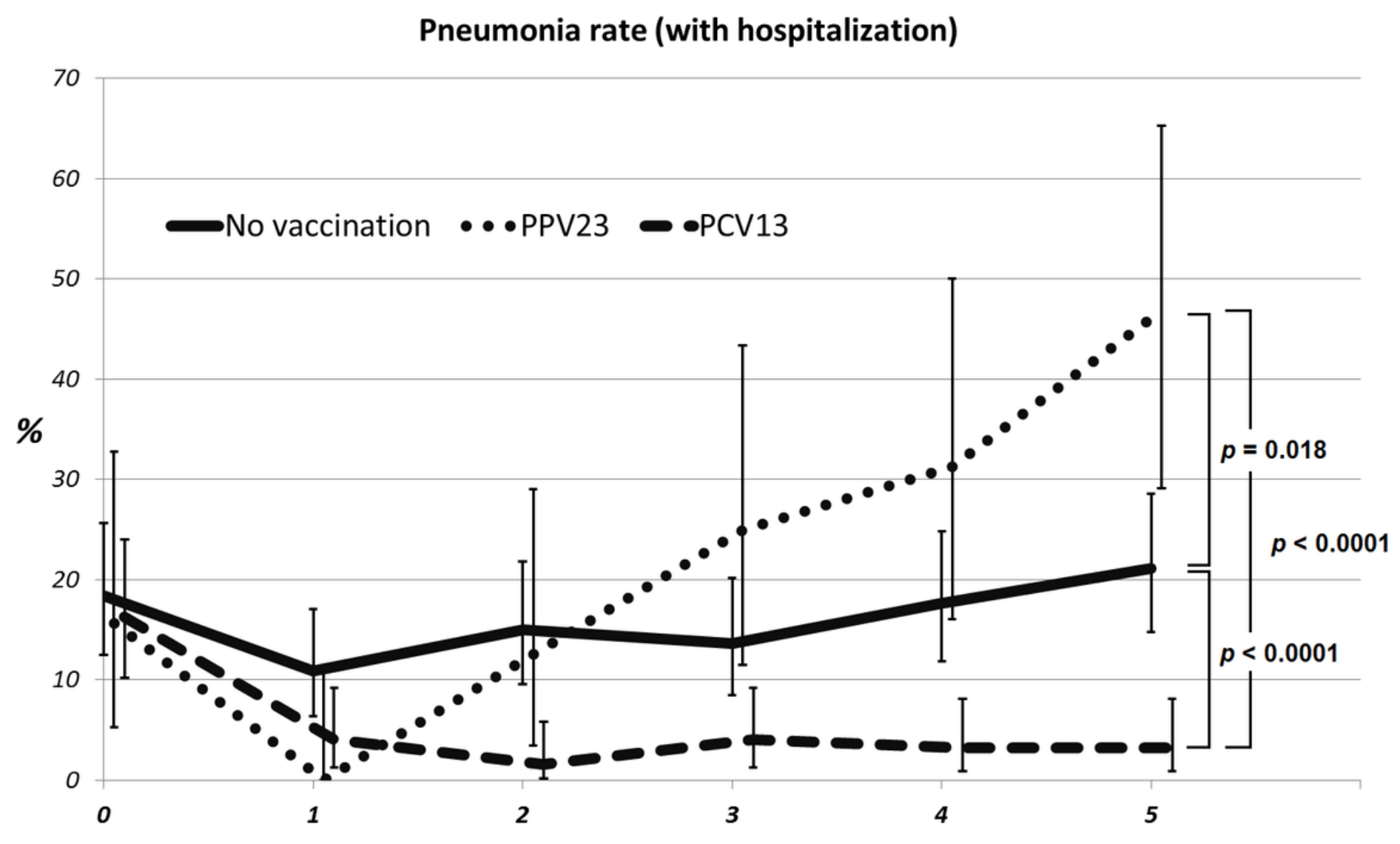

Follow-up, years after vaccination

Figure 1

The dynamics of pneumonia rate in the study groups. P value showed ANOVA statistics between groups. 


\section{COPD exacerbation rate}

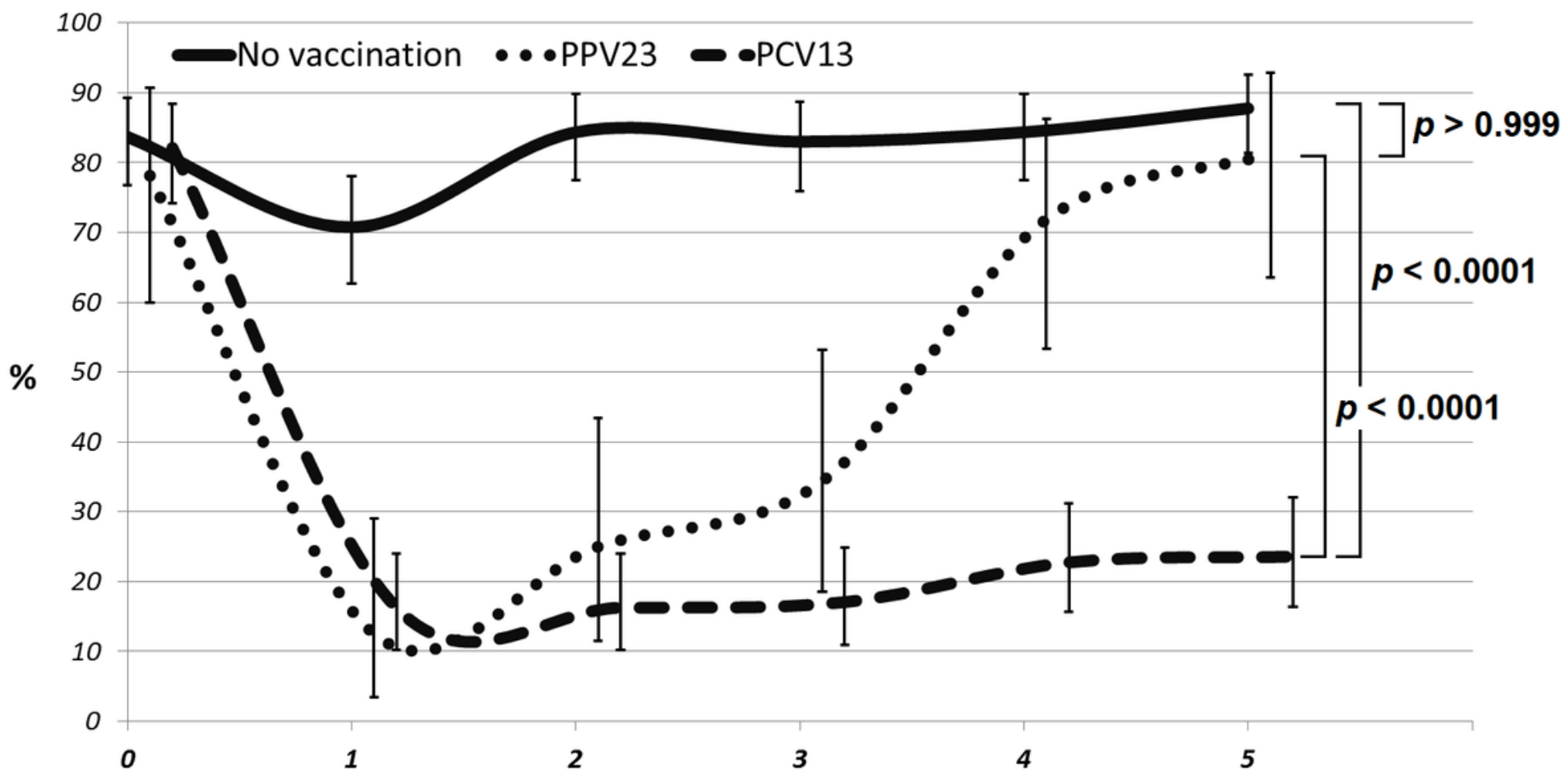

Follow-up, years after vaccination

Figure 2

The dynamics of COPD exacerbation rate in the study groups. P value showed ANOVA statistics between groups.

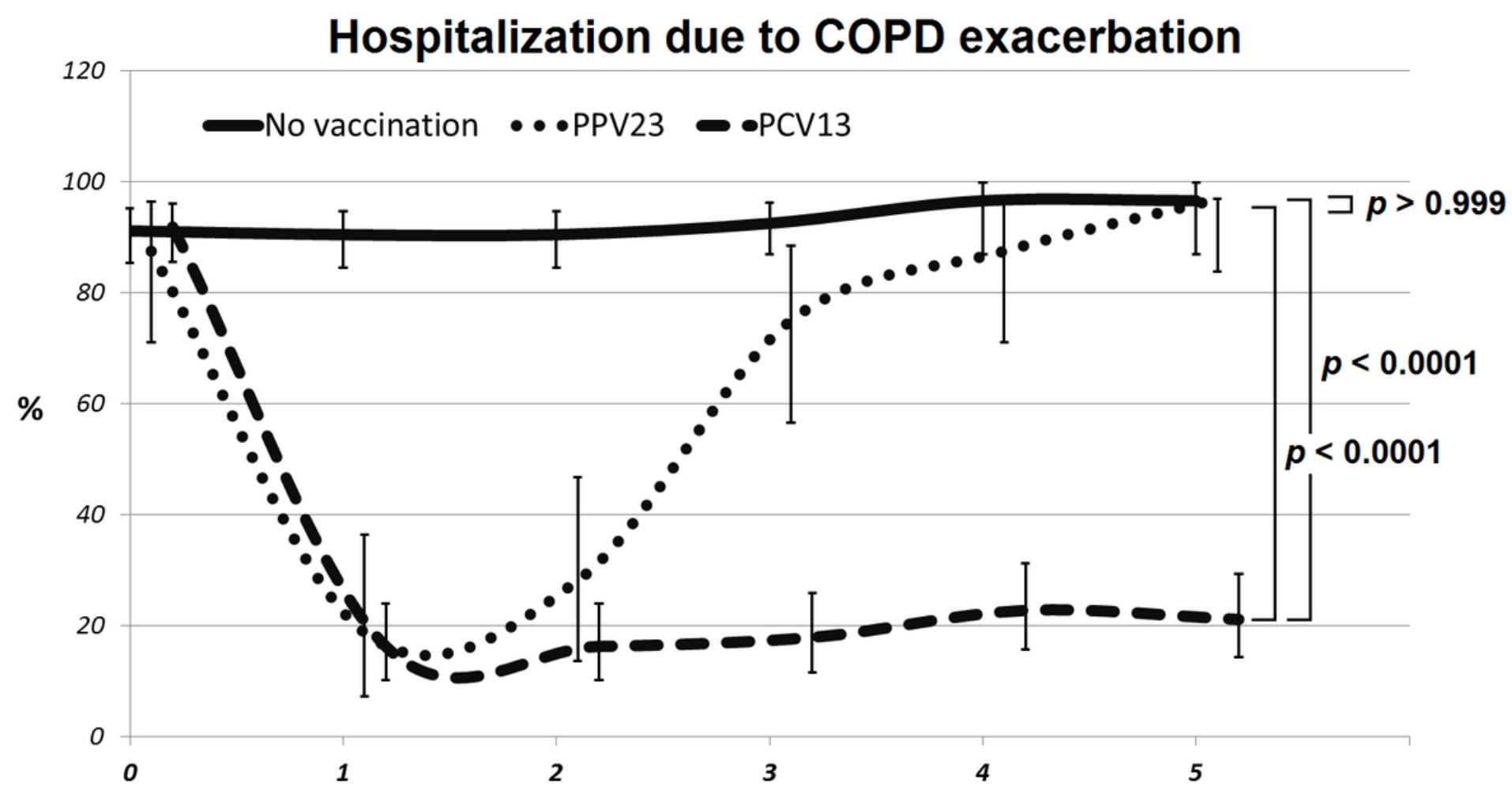

Follow-up, years after vaccination 
Figure 3

The dynamics of hospitalizations due to COPD exacerbation in the study groups. P value showed ANOVA statistics between groups.

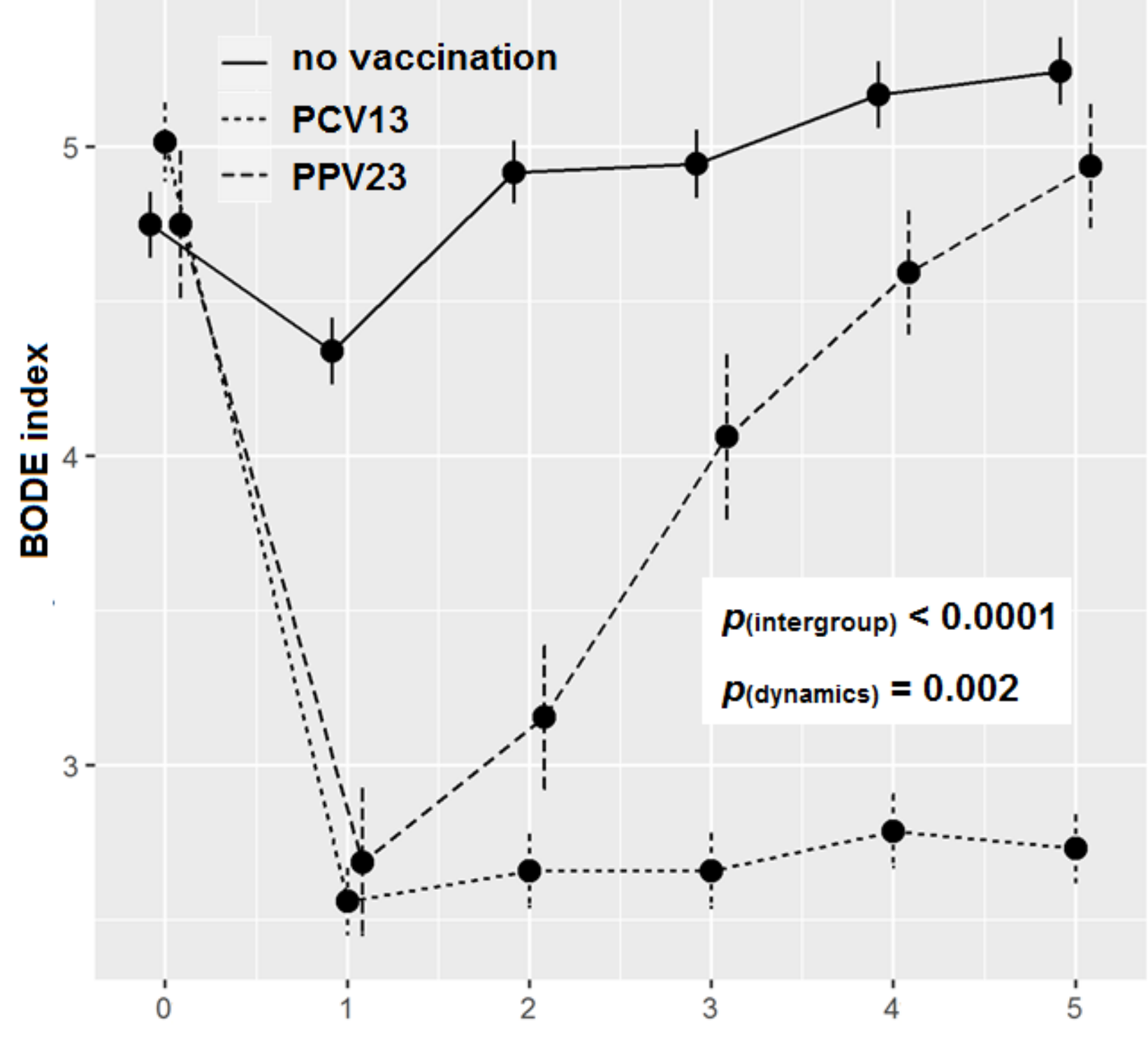

Follow-up, years after vaccination

Figure 4

Dynamics of BODE index in study groups during follow-up period. P value showed ANOVA statistics between groups. 


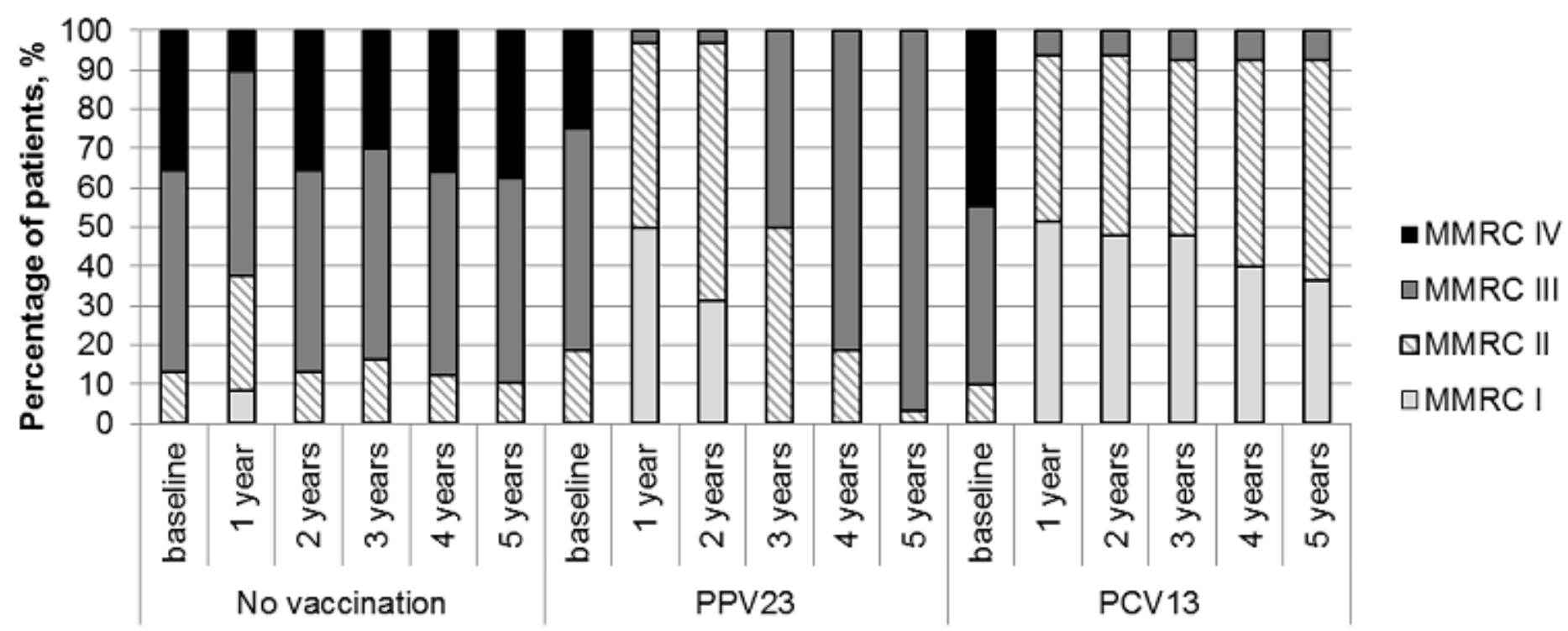

Figure 5

The dynamics of dyspnea severity (MMRC) in the study groups.
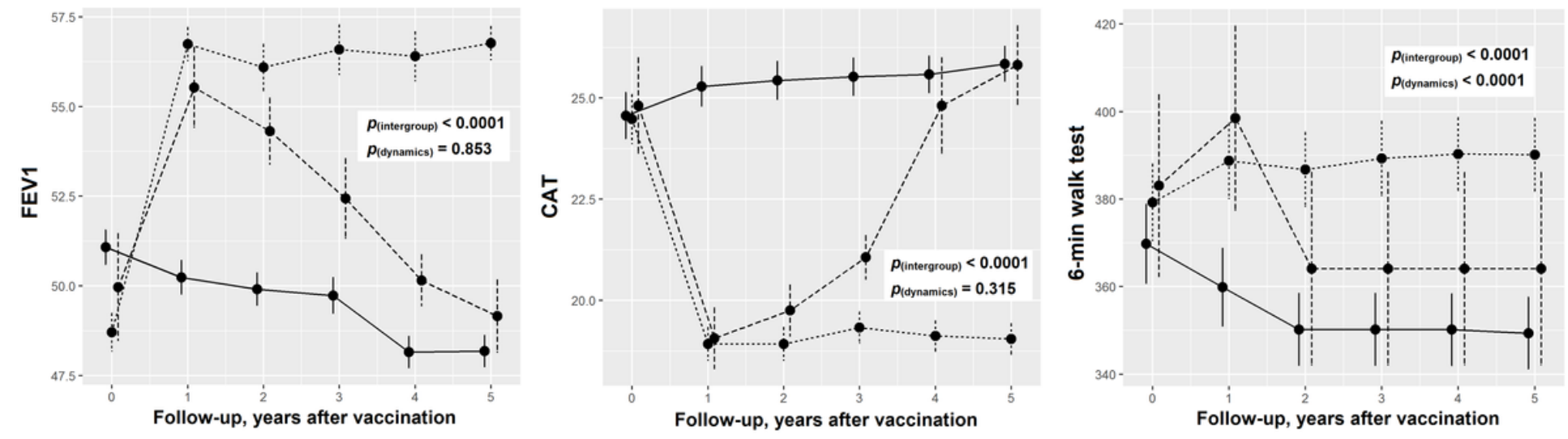

— No vaccination $\quad$-... PCV13 --. PPV23

Figure 6

The dynamics of FEV1, CAT, and 6-min walk test in the study groups. P value showed ANOVA statistics between groups. 


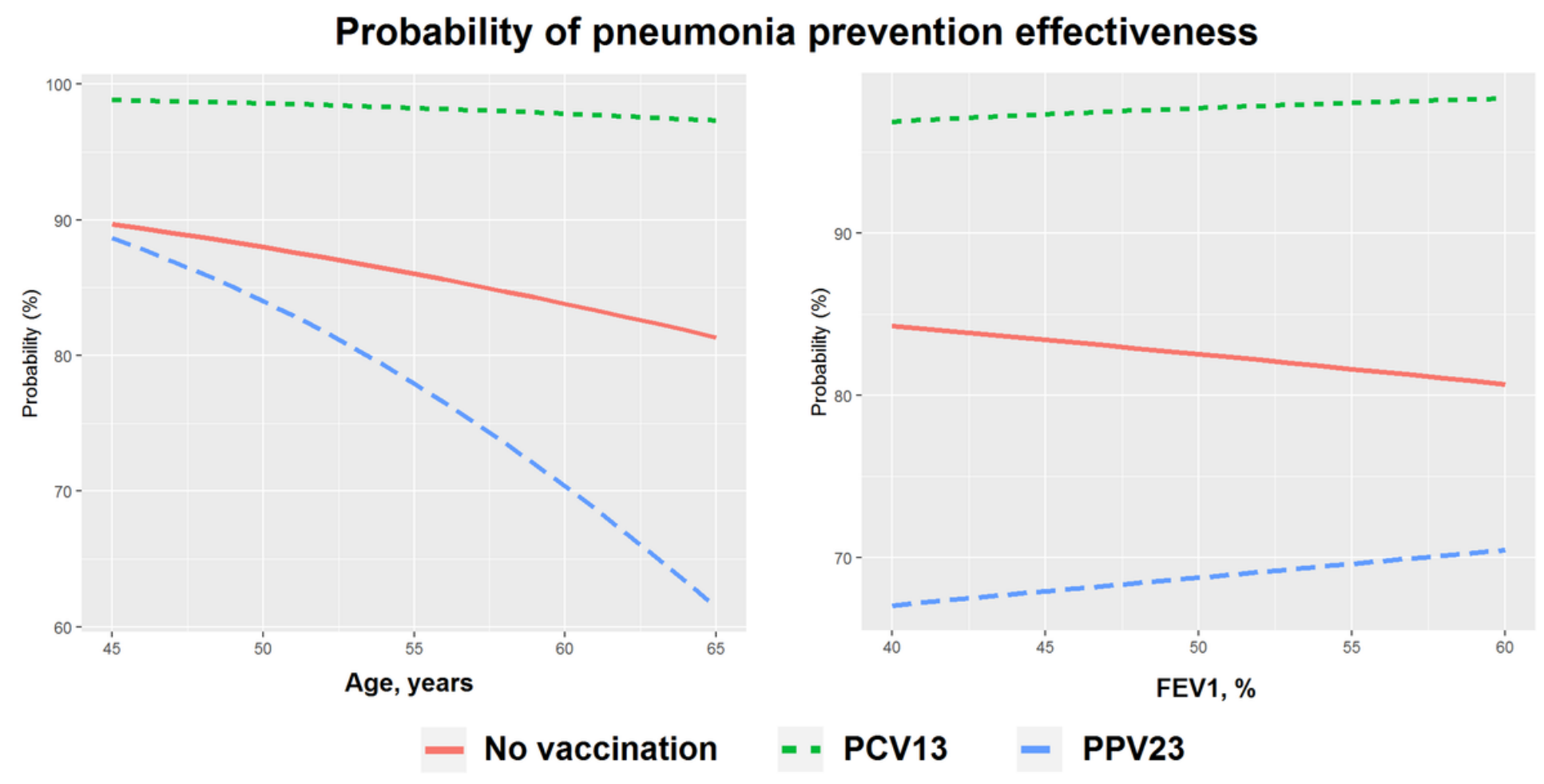

Figure 7

Multivariable model of probability of pneumonia prevention effectiveness. 